\title{
Geldanamycin induces apoptosis in human gastric carcinomas by affecting multiple oncogenic kinases that have synergic effects with TNF-related apoptosis-inducing ligand
}

\author{
HUI CHEN, LIANG-QING LI and DUN PAN \\ Department of Gastrointestinal Surgery, The First Clinical Medical College, Fujian Medical University, \\ Fuzhou, Fujian 350005, P.R. China
}

Received December 18, 2014; Accepted September 14, 2015

DOI: $10.3892 / \mathrm{ol} .2015 .3807$

\begin{abstract}
The aim of the present study was to evaluate the effect of geldanamycin (GA) on the treatment of human gastric carcinomas and to investigate the molecular mechanism that provides the basis for the combination of GA with the tumor necrosis factor (TNF)-related apoptosis-inducing ligand (TRAIL) induction strategy. The expression of target proteins at the mRNA level was determined using reverse transcription-polymerase chain reaction (RT-PCR), and apoptosis was evaluated with the terminal deoxynucleotidyl transferase mediated digoxigenin-dUTP nick-end labeling and Annexin V/propidium iodide (PI) staining methods. Phosphorylation of targeted kinases was studied using immunocytochemistry methods, and malignant phenotypes were studied using in vitro assays. GA treatment inhibits proliferation, migration and invasion, and induces apoptosis in human gastric cancer SGC-7901 cells, most likely by decreasing the expression of B-RAF and by phosphorylation of protein kinase B (AKT) and ERK. The inhibitory role of AKT in TRAIL regulation holds considerable potential for achieving a synergic effect in clinical therapy, using a combination of GA treatment and TRAIL induction. The present study provides a basis for the future application of heat shock protein 90 (Hsp90) inhibitors, such as GA, in the clinical treatment of gastric cancer, particularly in combination therapies with TRAIL inducers.
\end{abstract}

\section{Introduction}

Previously, studies have suggested that the chaperone heat shock protein 90 (Hsp90) is a promising target for cancer

Correspondence to: Dr Hui Chen, Department of Gastrointestinal Surgery, The First Clinical Medical College, Fujian Medical University, 20 Chazhong Road, Taijiang, Fuzhou, Fujian 350005, P.R. China

E-mail: huichen66@yeah.net

Key words: heat shock protein 90, gastric cancer, TNF-related apoptosis-inducing ligand, geldanamycin, protein kinase, apoptosis, synergic effect therapy (1-4). According to previous studies, the constitutive expression of Hsp90 was found to be 2-10 folds higher in malignant cells compared with normal cells (5). Additionally, several oncogenic proteins are dependent on Hsp90 to function, including B-RAF, protein kinase B (AKT) and extracellular signal-related kinase (ERK) (6). Inhibition of Hsp90 results in the dissociation of corresponding client proteins and induction of apoptosis in cancer cells, and may be accompanied by a reduction in chemotherapy resistance (7). Overall, these results highlight the crucial role of Hsp90 in the survival and growth of cancer cells and hold considerable potential for the development of small molecular inhibitors in drug treatment.

As an essential chaperone in vivo, Hsp90 assists a vast variety of client proteins, which may be categorized into three groups: i) Protein kinases, including B-RAF, AKT and ERK; ii) transcriptional factors or polymerases; and iii) miscellaneous groups (8). Numerous Hsp90-associated proteins are important in the occurrence and development of cancer. By forming complexes with Hsp90, RAF is stabilized, consequently inhibiting proteasome-dependent protein degradation (9). Disruption of Hsp90 function may significantly interfere with the RAF signaling pathway (10). In addition, AKT pathways participate in the regulation of cellular proliferation and apoptosis $(11,12)$. Previous studies have suggested that the occupancy of an Hsp90 binding site by ansamycins can result in a reduced half-life of AKT in vivo $(8,13)$. Similarly, ERK is closely involved in the pathogenesis of cancer and neurological disease $(14,15)$. Previous studies indicate that Hsp90 inhibitors may reduce the phosphorylation of ERK, without affecting the expression at mRNA and protein levels $(16,17)$. These oncogenic protein kinases contribute to cell proliferation, which is associated with apoptosis induced by Hsp90 inhibition.

TNF-related apoptosis-inducing ligand (TRAIL) is a cytokine produced and secreted by the majority of normal tissue cells (18). By binding to the death receptors DR4 and DR5, TRAIL causes apoptosis in cancer cells. For this reason, TRAIL has been an anti-cancer therapy target since the mid-1990s; however, no significant survival benefits have been found. Previous studies have reported that TRAIL can bind to and therefore activate $\mathrm{NF}-\kappa \mathrm{B}$, leading to the transcription of genes known to antagonize the death signaling pathway (19). 
Studies have also indicated that activation of the AKT survival pathway contributes to TRAIL resistance in cancer cells (20). These results suggest that the combination of oncogenic kinase inhibitors, such as AKT and TRAIL demonstrate promise in the induction of apoptosis.

Gastric cancer is the second most common cause of cancer-associated mortality. More importantly, gastric cancer is characterized by extremely low survival rates among patients (21). Numerous studies have suggested the importance of Hsp90 in the progression of gastric cancer (22-24). Certain small molecular inhibitors of Hsp90, including MPC-3100 (Myriad Pharmaceuticals, Salt Lake City, UT, USA), have demonstrated potential in the treatment of gastric cancer (25). Geldanamycin (GA) is a naturally occurring benzoquinone ansamycin that specifically binds to and inhibits Hsp90 (26). GA inhibits proliferation and induces apoptosis in certain types of cancer, including leukemia, colon cancer and breast cancer (27). However, the effect of GA on gastric cancer is unclear. In the present study, a systematic evaluation of GA in the treatment of gastric cancer was performed and the molecular mechanism behind Hsp90 inhibition was investigated. GA was found to significantly decrease the expression and phosphorylation of numerous oncogenic protein kinases, including AKT, which holds promise as a drug treatment, when used in combination with apoptosis-inducing TRAIL.

\section{Materials and methods}

Cell line and reagents. The human gastric cancer cell line SGC-7901 was purchased from Cell Bank of the Chinese Academy of Sciences (Shanghai, China). Monoclonal mouse anti-human antibodies against phosphorylated-AKT (p-AKT; $100 \mu \mathrm{l}$; cat no. 5106S) and phosphorylated-ERK (p-ERK; $200 \mu \mathrm{l}$; cat no. 9106S) were purchased from Cell Signaling Technology (Beverly, MA, USA). Annexin V-fluorescein isothiocyanate (FITC) Apoptosis Detection kit was purchased from Sigma-Aldrich (St. Louis, MO, USA). In-Situ Cell Death Detection kit was obtained from Roche (Mannheim, Germany). Dulbecco's modified Eagle's medium (DMEM) and fetal bovine serum (FBS) were purchased from Thermo Fisher Scientific (Waltham, MA, USA).

Cell culture of the SGC-7901 cancer cell line. Human gastric cancer SGC-7901 cells were cultured in DMEM containing $10 \%$ FBS, $1 \%$ glutathione (Sigma-Aldrich) and 1\% penicillin/streptomycin (Hyclone; GE Healthcare Life Sciences, Logan, UT, USA), at $37^{\circ} \mathrm{C}$ in a humidified atmosphere containing $5 \% \mathrm{CO}_{2}$. For all experiments, the cells were used in the exponential growth phase.

In vitro migration and invasion assays. An in vitro cell migration assay was performed with Millicell chambers (EMD Millipore, Bedford, MA, USA), as previously described (28). Briefly, the SGC-7901 cells were treated with $0.4 \mathrm{M}$ of GA for $48 \mathrm{~h}$, then harvested and suspended with serum-free medium. Then, $200 \mu \mathrm{l}$ of cell suspension $\left(2.5 \times 10^{5}\right.$ cells $\left./ \mathrm{ml}\right)$ was added to the upper chamber and $500 \mu 1$ NIH3T3-conditioned medium (Gibco; Thermo Fisher Scientific, Waltham, MA, USA) was added to the lower compartment. The chamber was incubated in $5 \% \mathrm{CO}_{2}$ at $37^{\circ} \mathrm{C}$ for $48 \mathrm{~h}$. The cells were removed from the upper chamber and the attached cells in the lower section were stained with $0.1 \%$ crystal violet. A similar procedure was performed for the invasion assay, but Matrigel (Clontech Laboratories, Inc., Mountain View, CA, USA) was added to the Transwell chambers (EMD Millipore) (28).

Terminal deoxynucleotidyl transferase mediated digoxigenin-dUTP nick-end labeling (TUNEL) assay. Cells were seeded in 6 -well plates $\left(1.5 \times 10^{5}\right.$ cells/well $)$ after placing a coverslip in each well. Apoptotic cells were detected using a TUNEL assay using the in situ cell death assay kit, according to the manufacturer's protocol. A BX53 microscope (Olympus, Tokyo, Japan) was used to count the total cell number and TUNEL-positive cell number in five fields of each TUNEL-stained section. The apoptotic index was calculated as the mean percentage of cells with definite positive TUNEL staining in the five microscopic fields.

Annexin V/PI staining assay. To evaluate the early stage of apoptosis induced by GA, an Annexin V/PI staining assay was performed. Subsequent to treatment with different concentrations of inhibitor for $48 \mathrm{~h}$, the SGC-7901 cells were harvested and washed three times with PBS. The cells were then stained with Annexin V/PI staining kit, as per the manufacturer's instructions. Flow cytometric analysis was immediately performed with a FACSCalibur Instrument (Becton Dickinson, Franklin Lakes, NJ, USA).

Immunocytochemistry assay. The SGC-7901 cells were treated with various concentrations of GA for $48 \mathrm{~h}$. The phosphorylation of AKT and ERK kinases was then evaluated by immunocytochemistry assay, as previously described (29).

Statistical analysis. The data were presented as the mean \pm standard deviation based on three independent experiments and statistically analyzed using a $t$-test or $\chi^{2}$ test. Analyses were performed using SPSS-13.0 statistical software (SPSS Inc., Chicago, IL, USA). P<0.05 was considered to indicate a statistically significant different.

\section{Results}

GA inhibits proliferation, migration and invasion of SGC-7901 cancer cells. By forming protein complexes, Hsp90 assists in maintaining the conformation, stability and function of certain oncogenic client proteins. Therefore, Hsp90 is important in cell proliferation, cell cycle progression, apoptosis and other features of malignant phenotypes, such as invasion, angiogenesis and metastasis $(2,4)$. To evaluate the potency of GA on gastric cancer, the effect of GA on the proliferation, migration and invasion of the gastric SGC-7901 cancer cell line was determined. The time taken for GA-induced proliferation inhibition is shown in Fig. 1A. GA significantly inhibits the proliferation of SGC-7901 cancer cells even at a low concentration of GA (0.2 M). The effect of GA on migration was studied using a Transwell assay. In this method, the cells that migrated through the pored membrane under the induction of NIH3T3-conditioned medium in the lower compartment were fixed and stained with crystal violet. The results revealed that treatment with $0.4 \mathrm{M}$ GA for $48 \mathrm{~h}$ significantly inhibits the 


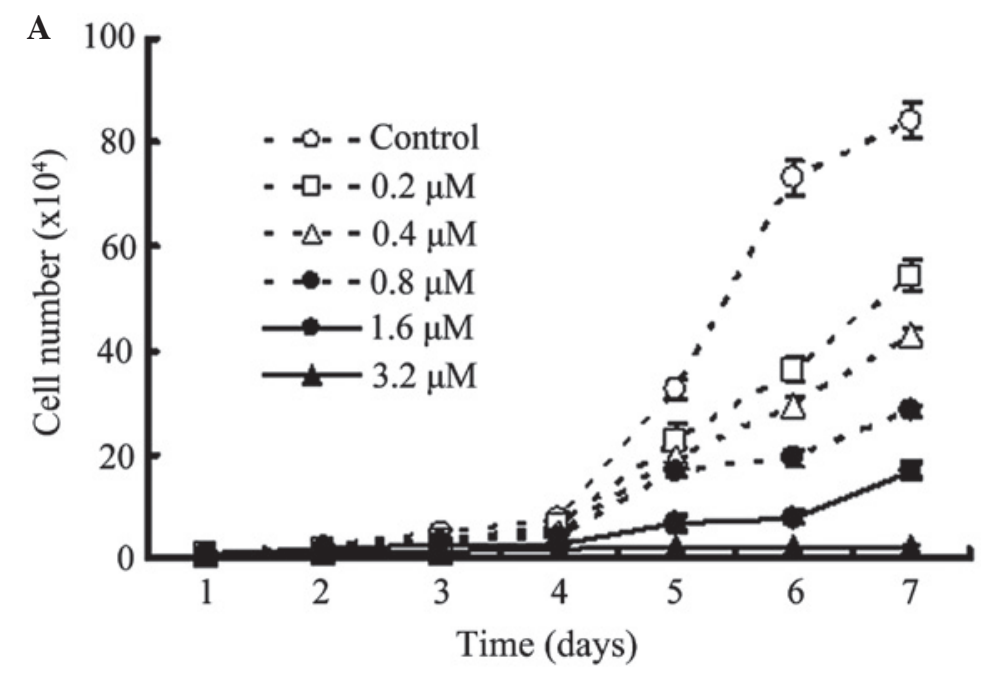

B

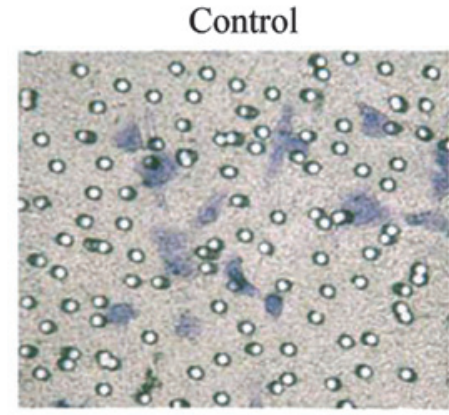

C

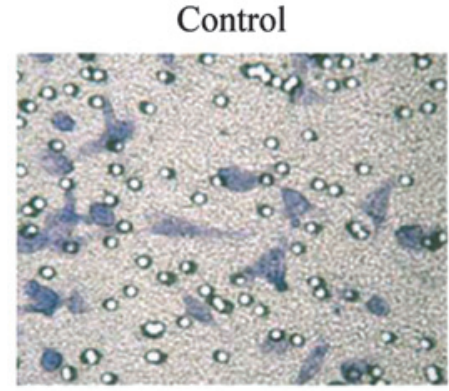

$0.4 \mu \mathrm{M} \mathrm{GA}$

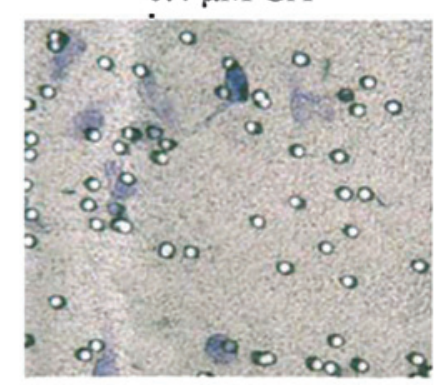

$0.4 \mu \mathrm{M} \mathrm{GA}$

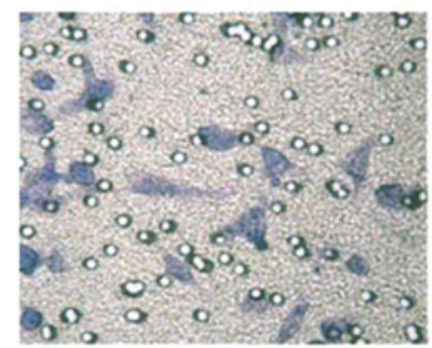

Figure 1. Treatment with GA inhibits the proliferation, invasion and migration of SGC-7901 cancer cells. (A) Dose-dependent effects of GA on cell proliferation at different time points. (B) The effect of GA on the migration of SGC-7901 cancer cells. (C) The effect of GA on the invasion of SGC-7901 cancer cells. GA, geldanamycin.

A

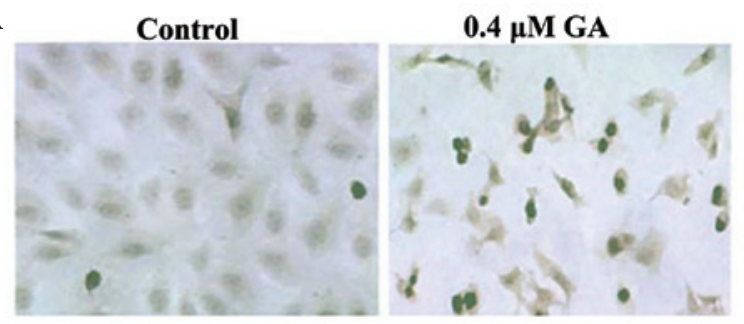

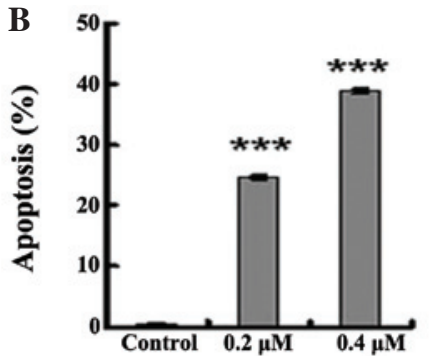

Figure 2. Treatment with GA-induced apoptosis of SGC-7901 cancer cells, as determined using (A) terminal deoxynucleotidyl transferase mediated digoxigen-dUTP nick-end labeling assay and (B) Annexin V-fluorescein isothiocyanate/propidium iodide staining assay, respectively $\left.{ }^{* * *} \mathrm{P}<0.001\right)$. GA, gelanamycin.

migration of SGC-7901 cells (Fig. 1B). The effect on invasion was also evaluated using a Matrigel invasion assay, where cells invade the Matrigel with the induction of NIH3T3-conditioned medium. Following incubation at $37^{\circ} \mathrm{C}$ for $48 \mathrm{~h}$, the cells invading the surface of the filter were fixed and stained using crystal violet. The treatment with $0.4 \mathrm{M} \mathrm{GA}$ was found to significantly inhibit the invasion of SGC-7901 cells (Fig. 1C).

GA induces apoptosis of SGC-7901 cancer cells. The effect of GA on SGC-7901 cells was evaluated using TUNEL assay and 


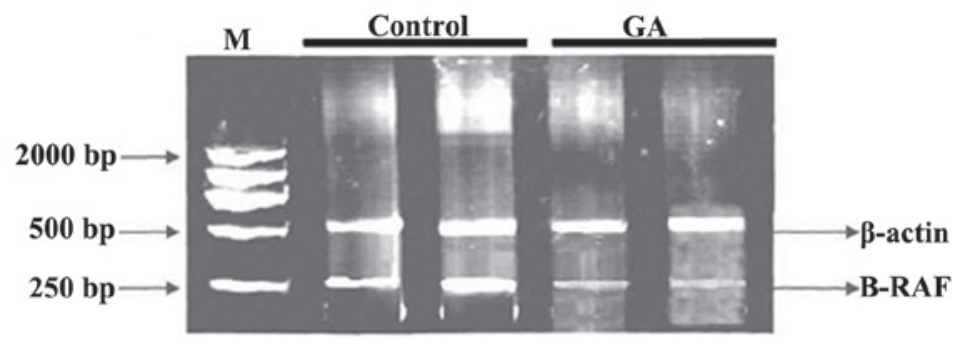

Figure 3. Treatment with 0.4 M GA decreased the mRNA level of B-RAF, determined using reverse transcriptase-polymerase chain reaction. GA, geldanamycin.
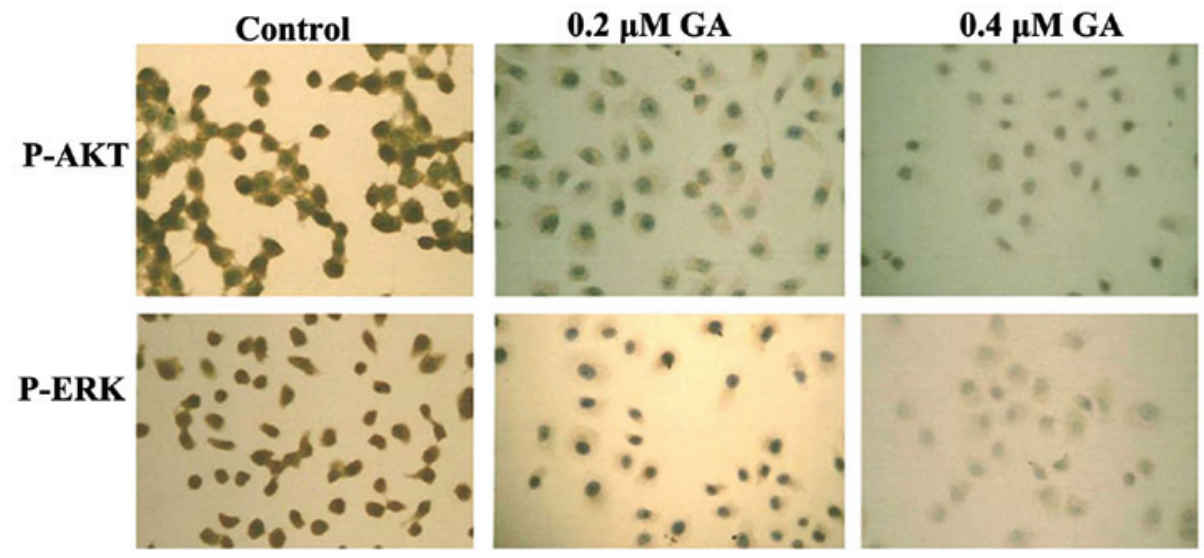

Figure 4. Treatment with GA decreased the phosphorylation level of AKT and ERK, determined using immunocytochemistry assays. GA, geldanamycin; ERK, extracellular signal-regulated kinase; AKT, protein kinase B.

Annexin V-FITC/PI staining assays. Cells undergoing apoptosis were indicated by brown granules in the nucleus. Treatment with 0.4 M GA induced apoptosis in SGC-7901 cancer cells compared with control cells (Fig. 2A). Similar results were observed by Annexin V-FITC/PI staining. GA significantly increased the percentage of apoptosis in SGC-7901 cancer cells at various concentrations $(0.2$ and $0.4 \mathrm{M})$ compared with control cells $(\mathrm{P}<0.001)$ (Fig. 2B).

$G A$ treatment decreases the expression of $B-R A F$. Most frequently found in melanoma and other types of cancer, mutation in B-RAF may cause B-RAF activation, thus facilitating its binding to the Hsp90-cdc37 complex that is essential for stability as well as function. Hsp90 inhibitors, such as 17-N-allylamino-17-demethoxygeldanamycin (17-AAG), stimulate B-RAF degradation in cancer cells $(30,31)$. When the B-RAF mRNA levels were measured using RT-PCR, it was found that treatment with $\mathrm{GA}(0.4 \mathrm{M})$ for $48 \mathrm{~h}$ induced a significant reduction of B-RAF mRNA in SGC-7901 cells (Fig. 3).

GA treatment decreases the phosphorylation of AKT and ERK. AKT and ERK can each promote cell survival and growth, as well as suppress cell apoptosis. The two kinases are considered to be involved in apoptosis induced by the inhibition of Hsp90. In the present study, the phosphorylation levels of two protein targets in GA-treated SGC-7901 cancer cells were determined with immunocytochemistry assays. Treatment with GA $(0.2$ and $0.4 \mathrm{M})$ significantly decreases the phosphorylation of AKT and ERK (Fig. 4). This is a notable result regarding the well-established role of activated AKT in the resistance of TRAIL in cancer cells, and holds great promise for the combination therapy of GA and TRAIL in gastric cancer chemotherapy.

\section{Discussion}

The molecular chaperone Hsp90 has been well-established as an important target of anticancer therapies. Hsp90 may stabilize and regulate the function of numerous client proteins, including kinases involved in cancer development $(2,32)$. The anticancer potential of chemical inhibitors of Hsp90, including GA and 17-AAG, has been extensively and comprehensively validated with cell models, xenografts and clinical studies $(6,27,30)$. The results of the present study revealed that GA strongly inhibits the proliferation of gastric cancer SGC-7901 cells. In addition, GA significantly increases the apoptosis of SGC-7901 cells, which supports the anticancer potential of GA.

As protein clients of Hsp90, B-RAF, AKT and ERK kinases are regarded to be involved in apoptosis induced by Hsp90 inhibitors $(33,34)$. The expression of B-RAF and the phosphorylation of AKT and ERK were determined. As a result, mRNA levels of B-RAF were found to be largely reduced in the GA-treated SGC-7901 cancer cells compared with control cells. By contrast, the phosphorylation of AKT and ERK significantly decreased in GA-treated SGC-7901 cells at various concentrations. These results suggest that the inhibition of oncogenic signaling pathways, such as the PI3K-AKT and Ras-RAF-MEK-ERK pathways, may contribute to apoptosis 
induced by GA in SGC-7901 cells. The present study provides a potential mechanism for the drug treatment of gastric cancer with GA. As previously mentioned, numerous studies have highlighted the inhibitory role of AKT in TRAIL signaling $(20,35)$. Down-regulation of constitutively active AKT may lead to the resistance of cancer cells to recombinant human (rh)TRAIL. During this process, Hsp90 protects cancer cells from apoptosis by stabilizing AKT and NF- $\mathrm{kB}$. Therefore, inhibition of Hsp90 may result in cell cycle arrest and more importantly, apoptosis $(36,37)$. The use of 17-AAG as an Hsp90 inhibitor is currently being clinically evaluated in phase I trials $(38,39)$.

In conclusion, combinations of Hsp90 inhibitors with TRAIL inducers exhibited synergistic effects on apoptosis induction in gastric cancer. Thus, the present study provides a rationale for the combined use of GA and TRAIL in the clinic.

\section{Acknowledgements}

The present study was supported by the National Key Clinical Specialist Construction Project of China.

\section{References}

1. Solit DB and Rosen N: Hsp90: A novel target for cancer therapy. Curr Top Med Chem 6: 1205-1214, 2006.

2. Maloney A and Workman P: HSP90 as a new therapeutic target for cancer therapy: The story unfolds. Expert Opin Biol Ther 2: $3-24,2002$

3. Mahalingam D, Swords R, Carew JS, Nawrocki ST, Bhalla K and Giles FJ: Targeting HSP90 for cancer therapy. Br J Cancer 100 $1523-1529,2009$

4. Sidera K and Patsavoudi E: HSP90 inhibitors: Current development and potential in cancer therapy. Recent Pat Anticancer Drug Discov 9: 1-20, 2014.

5. Ferrarini M, Heltai S, Zocchi MR and Rugarli C: Unusual expression and localization of heat-shock proteins in human tumor cells. Int J Cancer 51: 613-619, 1992.

6. Neckers L and Neckers K: Heat-shock protein 90 inhibitors as novel cancer chemotherapeutic agents. Expert Opin Emerg Drugs 7: 277-288, 2002.

7. Connell P, Ballinger CA, Jiang J, Wu Y, Thompson LJ, Höhfeld J and Patterson C: The co-chaperone CHIP regulates protein triage decisions mediated by heat-shock proteins. Nat Cell Biol 3 : 93-96, 2001.

8. Goetz MP, Toft DO, Ames MM and Erlichman C: The Hsp90 chaperone complex as a novel target for cancer therapy. Ann Oncol 14: 1169-1176, 2003.

9. Schulte TW, Blagosklonny MV, Ingui $\mathrm{C}$ and Neckers L: Disruption of the Raf-1-Hsp90 molecular complex results in destabilization of Raf-1 and loss of Raf-1-Ras association. J Bio Chem 270: 24585-24588, 1995

10. Schulte TW, Blagosklonny MV, Romanova L, Mushinski JF, Monia BP, Johnston JF, Nguyen P, Trepel J and Neckers LM: Destabilization of Raf-1 by geldanamycin leads to disruption of the Raf-1-MEK-mitogen-activated protein kinase signalling pathway. Mol Cell Biol 16: 5839-5845, 1996.

11. Neri LM, Borgatti P, Capitani S and Martelli AM: The nuclear phosphoinositide 3-kinase/AKT pathway: A new second messenger system. Biochim Biophys Acta 1584: 73-80, 2002

12. Kumar A, Rajendran V, Sethumadhavan R and Purohit R: AKT kinase pathway: A leading target in cancer research. ScientificWorldJournal 2013: 756134, 2013

13. Basso AD, Solit DB, Chiosis G, Giri B, Tsichlis P and Rosen N: Akt forms an intracellular complex with heat shock protein 90 (Hsp90) and Cdc37 and is destabilized by inhibitors of Hsp90 function. J Biol Chem 277: 39858-39866, 2002.

14. Ma W and Quirion R: The ERK/MAPK pathway, as a target for the treatment of neuropathic pain. Expert Opin Ther Targets 9: 699-713, 2005.

15. Lee JT Jr and McCubrey JA: The Raf/MEK/ERK signal transduction cascade as a target for chemotherapeutic intervention in leukemia. Leukemia 16: 486-507, 2002.
16. Dou F, Yuan LD and Zhu JJ: Heat shock protein 90 indirectly regulates ERK activity by affecting Raf protein metabolism. Acta Biochim Biophys Sin (Shanghai) 37: 501-505, 2005.

17. Johnstone RW, Frew AJ and Smyth MJ: The TRAIL apoptotic pathway in cancer onset, progression and therapy. Nat Rev Cancer 8: 782-798, 2008.

18. Wiley SR, Schooley K, Smolak PJ, Din WS, Huang CP, Nicholl JK, Sutherland GR, Smith TD, Rauch C, Smith CA, et al: Identification and characterization of a new member of the TNF family that induces apoptosis. Immunity 3: 673-682, 1995.

19. Song JJ and Lee YJ: Differential cleavage of Mst1 by caspase-7/-3 is responsible for TRAIL-induced activation of the MAPK superfamily. Cell Signal 20: 892-906, 2008.

20. Xu J, Zhou JY, Wei WZ and Wu GS: Activation of the Akt survival pathway contributes to TRAIL resistance in cancer cells. PloS One 5: e10226, 2010.

21. Roder DM: The epidemiology of gastric cancer. Gastric Cancer 5 (Suppl): 5-11, 2002.

22. Chen M, Xu J and Zhao J: Effects of HSP90 inhibitor 17-AAG on cell cycle and apoptosis of human gastric cancer cell lines SGC-7901. Nan Fang Yi Ke Da Xue Xue Bao 33: 271-275, 2013 (In Chinese).

23. Wainberg ZA, Anghel A, Rogers AM, Desai AJ, Kalous O, Conklin D, Ayala R, O'Brien NA, Quadt C, Akimov M, et al: Inhibition of HSP90 with AUY922 induces synergy in HER2-amplified trastuzumab-resistant breast and gastric cancer. Mol Cancer Ther 12: 509-519, 2013.

24. Redlak MJ and Miller TA: Targeting PI3K/Akt/HSP90 signaling sensitizes gastric cancer cells to deoxycholate-induced apoptosis. Dig Dis Sci 56: 323-329, 2011.

25. Hede K: Gastric cancer: Trastuzumab trial results spur search for other targets. J Natl Cancer Inst 101: 1306-1307, 2009.

26. Stebbins CE, Russo AA, Schneider C, Rosen N, Hartl FU and Pavletich NP: Crystal structure of an Hsp90-geldanamycin complex: Targeting of a protein chaperone by an antitumor agent. Cell 89: 239-250, 1997.

27. Supko JG, Hickman RL, Grever MR and Malspeis L: Preclinical pharmacologic evaluation of geldanamycin as an antitumor agent. Cancer Chemother Pharmacol 36: 305-315, 1995.

28. Ma Q, Jiang Q, Pu Q, Zhang X, Yang W, Wang Y, Ye S, Wu S, Zhong G, Ren J, et al: MicroRNA-143 inhibits migration and invasion of human non-small-cell lung cancer and its relative mechanism. Int J Biol Sci 9: 680-692, 2013.

29. Köhrmann A, Kammerer U, Kapp M, Dietl J and Anacker J: Expression of matrix metalloproteinases (MMPs) in primary human breast cancer and breast cancer cell lines: New findings and review of the literature. BMC Cancer 16: 188, 2009.

30. da Rocha Dias S, Friedlos F, Light Y, Springer C, Workman P and Marais R: Activated B-RAF is an Hsp90 client protein that is targeted by the anticancer drug 17-allylamino-17-demethoxygeldanamycin. Cancer Res 65: 10686-10691, 2005.

31. Grbovic OM, Basso AD, Sawai A, Ye Q, Friedlander P, Solit D and Rosen N: V600E B-Raf requires the Hsp90 chaperone for stability and is degraded in response to Hsp90 inhibitors. Proc Natl Acad Sci USA 103: 57-62, 2006.

32. Neckers L and Neckers K: Heat-shock protein 90 inhibitors as novel cancer chemotherapeutics - an update. Expert Opin Emerg Drugs 10: 137-149, 2005.

33. Zhang $\mathrm{H}$ and Burrows F: Targeting multiple signal transduction pathways through inhibition of Hsp90. J Mol Med (Berl) 82: 488-499, 2004

34. Koga F, Xu WP, Karpova TS, McNally JG, Baron R and Neckers L: Hsp90 inhibition transiently activates Src kinase and promotes Src-dependent Akt and Erk activation. Proc Natl Acad Sci USA 103: 11318-11322, 2006.

35. Matsuoka T and Yashiro M: The role of PI3K/Akt/mTOR signaling in gastric carcinoma. Cancers (Basel) 6: 1441-1463, 2014.

36. Hostein I, Robertson D, DiStefano F, Workman P and Clarke PA: Inhibition of signal transduction by the Hsp90 inhibitor 17-Allylamino-17- demethoxygeldanamycin results in cytostasis and apoptosis. Cancer Res 61: 4003-4009, 2001.

37. Powers MV, Clarke PA and Workman P: Dual targeting of HSC70 and HSP72 inhibits HSP90 function and induces tumor-specific apoptosis. Cancer Cell 14: 250-262, 2008.

38. Kim YS, Alarcon SV, Lee S, Lee MJ, Giaccone G, Neckers L and Trepel JB: Update on Hsp90 inhibitors in clinical trial. Curr Top Med Chem 9: 1479-1492, 2009.

39. Jhaveri K, Taldone T, Modi S and Chiosis G: Advances in the clinical development of heat shock protein 90 (Hsp90) inhibitors in cancers. Biochim Biophys Acta 1823: 742-755, 2012. 